\title{
ILLUMINATING ELECTRONICS PROBLEM SOLVING WITH THE CAL POLY MOHAT TECHNIQUE
}

\author{
David Braun ${ }^{1}$, Fred DePiero ${ }^{2}$ and Michael Borland ${ }^{3}$
}

\begin{abstract}
This paper describes a Cal Poly version of circuit analysis to assist both students and instructors. The primary focus of the technique is helping students perform hand analysis, particularly the type of hand analysis that clarifies the student's understanding of circuit operation in a manner that can benefit subsequent design decisions. The technique nicely complements graphical solution techniques such as load-line analyses and computer aided circuit simulation. MoHAT, an abbreviation of Model-HypothesizeAnalyze-Test, provides students with a roadmap to use, when analyzing even relatively complex circuits containing diodes and transistors. This paper emphasizes the pedagogy of MoHAT and describes a web-based software tool developed to help students visualize circuit operation.
\end{abstract}

Index Terms - Electronics Pedagogy, Circuit Analysis Software, Web-based Tools.

This presentation describes the pedagogy of MoHAT, short for Model-Hypothesize-Analyze-Test, a Cal Poly version of circuit analysis that assists both students and instructors. Analysis of circuits containing non-linear elements benefits from the use of equivalent circuit models. For this reason, introductory electronics and circuit analysis textbooks often outline a strategy for problem solving and suggest that students apply equivalent circuit models[1]-[2]. The MoHAT approach appeals to students' desire for useful and widespread application while allowing instructors to insert the technique easily into courses during passive lectures, during active learning exercises, and during homework assignments. Instructors at Cal Poly apply the MoHAT approach primarily to Sophomore and Junior level electronics lecture and laboratory courses.

The MoHAT technique consists of four steps:

a) Select an appropriate circuit Model for each circuit element.

b) Hypothesize the mode of operation for each circuit element.

c) Apply circuit analysis methods to Analyze the operation of the equivalent circuit.

d) Test results against hypotheses and iterate if necessary to achieve self-consistent results.

The primary focus of the technique is helping students perform hand analysis, particularly the type of hand analysis that clarifies the student's understanding of circuit operation in a manner that can benefit subsequent design decisions. The technique nicely complements graphical solution techniques such as load-line analyses and computer aided circuit simulation. The technique provides students with a roadmap to use, when analyzing even relatively complex circuits containing diodes and transistors. MoHAT is an effective technique, because it generalizes well to a wide variety of circuits and because it is easy for students to learn.

This presentation also introduces a web-based software tool developed to help students visualize circuit operation. Figure 1 shows an example screenshot from the MoHAT tool. From a PSpice[3] output file, with suffice ".out," the MoHAT tool generates a circuit schematic with color codes to indicate modes of transistor and diode operation. The legends in Figure 1 indicate the colors associated with the modes of operation for field effect and bipolar junction transistors. Figure 1 also contains graphs of transfer characteristics at two output nodes. The MoHAT tool is available on the web[4].

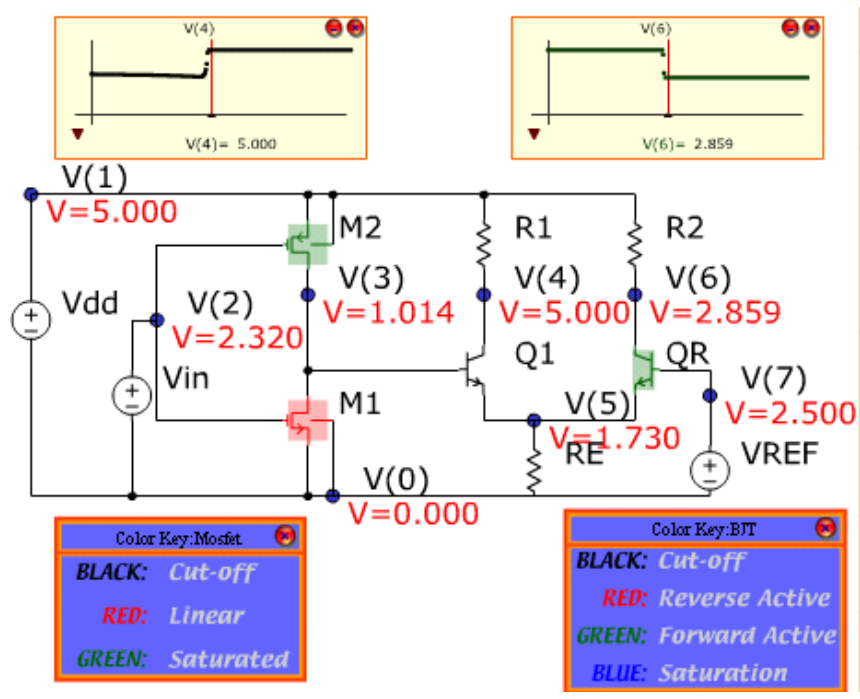

FIGURE 1

SCREENSHOT OF MOHAT ANALYSIS OF DC CIRCUIT OPERATION

\section{REFERENCES}

[1] Dorf, R. C. and Svoboda, J. A., Introduction to Electric Circuits, 4/e, 1999, John Wiley \& Sons, Inc. p. 23

[2] Alexander, C., Sadiku, M., Fundamentals of Electric Circuits, 2000, McGraw-Hill

[3] http://www.cadencepcb.com/products/downloads/PSpicestudent/

[4] http://www.mohat.net/

\footnotetext{
${ }^{1}$ David Braun, Cal Poly State University, Electrical Engineering Department, San Luis Obispo, CA 93407 dbraun@calpoly.edu

${ }^{2}$ Fred DePiero, Cal Poly State University, Electrical Engineering Department, San Luis Obispo, CA 93407 fdepiero@calpoly.edu

${ }^{3}$ Michael Borland, Cal Poly State University, Electrical Engineering Department, San Luis Obispo, CA 93407 mborland@alumni.calpoly.edu 